\title{
The Factorization of a Polynomial Defined by Partitions
}

\author{
R. P. Langlands
}

School of Mathematics, Institute for Advanced Study, Princeton, NJ 08540, USA

\begin{abstract}
Polynomials whose vanishing is necessary and sufficient for the existence of primary holomorphic conformal fields are introduced, and in certain cases decomposed into linear factors.
\end{abstract}

\section{Introduction}

It is best to work with unordered partitions. Thus if $k$ is a positive integer, a partition of length $r$ of the interval $[0, k]$ is a sequence, $0=k_{0}<k_{1}<\cdots<k_{r}=k$, of positive integers. Set $k_{1}^{\prime}=k-k_{i}$.

Fix $k$, and let $x, Y$, and $\Delta$ be three indeterminates. Form the polynomial $P_{k}(x, Y, \Delta)$ given by

$$
\sum_{\left\{k_{1}, \ldots, k_{r-1}\right\}} x^{k-r} \prod_{i=1}^{r}\left(k_{i}^{\prime}+Y+\Delta\left(k_{i}-k_{i-1}\right)\right)\left(\prod_{i=1}^{r-1} k_{i} k_{1}^{\prime}\right)^{-1} .
$$

In the summation $r$ is not fixed, so that the sum runs over all unordered partitions of $k$. The polynomial is of degree $k$ in $Y$, and the coefficient of $Y^{k}$ is $((k-1) !)^{-2}$.

It can be factored explicitly. For this it is convenient to write

$$
\Delta=h_{p, q}(m)=\frac{((m+1) p-m q)^{2}-1}{4 m(m+1)} .
$$

Observe that if $m \neq 0,-1$ then, given $\Delta$, this equation can always be solved for $p$ and $q$. Set

$$
\begin{aligned}
Y_{s}(m)= & \left(\left((1-k)^{2}-(p-q+s)^{2}\right) m^{2}+2((1-k)-(p-q+s) p) m+1-p^{2}\right) / 4 m(m+1) \\
= & h_{1, k}(m)-h_{p, q-s}(m), \\
Y_{r}^{\prime}(m)= & \left(\left((k-1)^{2}-(p-r-q)^{2}\right) m^{2}+2((k-1) k-(p-r-q)(p-r)) m\right. \\
& \left.+k^{2}-(p-r)^{2}\right) / 4 m(m+1) \\
= & h_{k, 1}(m)-h_{p-r, q}(m) .
\end{aligned}
$$




\section{Theorem 1.}

(a) If $m$ is defined by $x=-m /(m+1)$ and if $\Delta=h_{p, q}(m)$, then

$$
P_{k}(x, Y, \Delta)=((k-1) !)^{-2} \prod_{\substack{|s|<k \\ s \equiv k-1(\bmod 2)}}\left(Y-Y_{s}(m)\right) .
$$

(b) If $m$ is defined by $x=-(m+1) / m$ and if $\Delta=h_{p, q}(m)$, then

$$
P_{k}(x, Y, \Delta)=((k-1) !)^{-2} \prod_{\substack{|r|<k \\ r \equiv k-1(\bmod 2)}}\left(Y-Y_{r}^{\prime}(m)\right) .
$$

The purpose of this paper is to prove this theorem. As is explained in an appendix, it has its origins in the theory of the Virasoro algebra and conformally invariant quantum fields. Thanks are due to Y. Saint-Aubin for explaining to me the formulas (A.1), (A.2) proved by him and Benoit in [BSA], for they were the starting point of this paper, to W. Casselman for a number of conversations and, in particular, for checking the critical Identity 2.4 on the computer long before I was able to prove it, to Lyman Hurd for instruction in the use of the computer, and to W.-C. Hsiang for avuncular advice about the title.

\section{Simple Reductions}

The two parts of the theorem are equivalent. They are both stated, because they are both to be used in the Appendix. If $m^{\prime}=-m-1$ then

$$
\frac{m+1}{m}=\frac{m^{\prime}}{m^{\prime}+1}
$$

and

$$
h_{p, q}(m)=h_{q, p}\left(m^{\prime}\right)
$$

for all $p, q$. Thus upon interchange of $p$ and $q$ the expression $Y_{s}\left(m^{\prime}\right)$ becomes $Y_{s}^{\prime}(m)$.

It is enough to prove the first statement of the theorem. We begin with a sequence of observations, stated as lemmas. To indicate the dependence of $Y_{s}(m)$ on $k$ we write $Y_{s}^{k}(m)$.

\section{Lemma 2.1.}

$$
Y_{s}^{k+2}(m)=Y_{s}^{k}(m)-1-(k+1) x .
$$

The difference $Y_{s}^{k+2}(m)-Y_{s}^{k}(m)$ is seen upon inspection to be

$$
\frac{\left((k+1)^{2}-(k-1)^{2}\right) m^{2}-2((k+1)-(k-1)) m}{4 m(m+1)}=\frac{k m-1}{m+1}=\frac{(k+1) m}{m+1}-1 \text {. }
$$

Lemma 2.2. The product

$$
\left(Y-Y_{k+1}^{k+2}(m)\right)\left(Y-Y_{-k-1}^{k+2}(m)\right)
$$

is equal to

$$
(Y+\Delta)(Y+\Delta+k+1)+(k+1) x(Y+(k+2) \Delta) .
$$

The lemma is tantamount to two assertions: 


$$
\begin{aligned}
& Y_{k+1}^{k+2}(m)+Y_{-k-1}^{k+2}(m)=-2 \Delta-(k+1)-(k+1) x ; \\
& Y_{k+1}^{k+2}(m)+Y_{-k-1}^{k+2}(m)=\Delta(\Delta+k+1)+(k+1)(k+2) x \Delta .
\end{aligned}
$$

The sum on the left of the first identity is equal to

Since

$$
\left(-2(p-q)^{2} m^{2}-4(k+1) m-4(p-q) p m+2\left(1-p^{2}\right)\right) / 4 m(m+1) .
$$

$$
\Delta=\left((p-q)^{2} m^{2}+2(p-q) p m+p^{2}-1\right) / 4 m(m+1),
$$

the first equality is immediate.

For the second, one observes that the product on the left is

$$
\begin{gathered}
\left(\left((p-q)^{2} m^{2}+2((k+1)+(p-q) p) m+p^{2}-1\right)^{2}\right. \\
\left.-4(k+1)^{2}\left((p-q) m^{2}+p m\right)^{2}\right) / 16 m^{2}(m+1)^{2}
\end{gathered}
$$

and this is

$$
\left(\Delta+\frac{k+1}{2(m+1)}\right)^{2}-(k+1)^{2}(\Delta+1 / 4 m(m+1))(m / m+1) .
$$

The second equality follows.

\section{Lemma 2.3.}

(a) $P_{1}(x, Y, \Delta)=Y-Y_{0}^{1}(m)$,

(b) $P_{2}(x, Y, \Delta)=\left(Y-Y_{-1}^{2}(m)\right)\left(Y-Y_{1}^{2}(m)\right)$.

The first assertion is clear; the second follows from the previous lemma and a direct calculation of $P_{2}(x, Y, \Delta)$ as

$$
(Y+1+\Delta)(Y+\Delta)+x(Y+2 \Delta)
$$

Theorem 1(a) is thus seen to be equivalent to the validity of the following identity.

Identity 2.4. The polynomial $P_{k+2}(x, Y, \Delta)$ is equal to the product of

$$
(k(k+1))^{-2} P_{k}(x, Y+1+(k+1) x, \Delta)
$$

and

$$
(Y+\Delta)(Y+\Delta+k+1)+(k+1) x(Y+(k+2) \Delta) .
$$

Fix $r>-2$ and consider on both sides of the identity the coefficient of $x^{k-r}$. On the left it is $P_{k+2}^{r+2}(Y, \Delta)$, the sum over all partitions $\left\{k_{1}, \ldots, k_{r+1}\right\}$ of $[0, k+2]$ into $r+2$ intervals of the expression

$$
\prod_{i=1}^{r+2}\left(k_{i}^{\prime}+Y+\Delta\left(k_{i}-k_{i-1}\right)\right) \prod_{i=1}^{r+2}\left(k_{i} k_{i}^{\prime}\right)^{-1}
$$

Since $k_{i}-k_{i-1}=k_{i-1}^{\prime}-k_{i}^{\prime}$, this equals

$$
\prod_{i=1}^{r+2}\left(\Delta\left(Y+k_{i-1}^{\prime}\right)+(1-\Delta)\left(Y+k_{1}^{\prime}\right)\right) \prod_{i=1}^{r+1}\left(k_{i} k_{i}^{\prime}\right)^{-1} \text {. }
$$

If $S_{k}^{r}(Y+1, \Delta)$ is the coefficient of $x^{k-r}$ in (2.a) then the coefficient of $x^{k-r}$ on 
the right side of the identity is

$$
S_{k}^{r}(Y+1, \Delta)(Y+\Delta)(Y+\Delta+k+1)+S_{k}^{r+1}(Y+1, \Delta)(k+1)(Y+(k+2) \Delta) \text {. }
$$

We fix $k$ and $r$, and show that the coefficients $x^{k-r}$ on both sides of the identity are equal.

\section{Further Reductions}

To calculate $S_{k}^{r}(Y, \Delta)$ we have to expand the products

$$
x^{k-s} \prod_{i=1}^{s}\left(k_{i}^{\prime}+Y+(k+1) x+\Delta\left(k_{i}-k_{i-1}\right)\right),
$$

retaining only the coefficient of $x^{k-r}$. Thus we obtain a sum over all partitions of $k$ and over all subsets $\Sigma$ with $r$ elements of those partitions of

$$
(k+1)^{s-r} \prod_{i \in \Sigma}\left(k_{i}^{\prime}+Y+\Delta\left(k_{i-1}^{\prime}-k_{i}^{\prime}\right)\right) \prod_{i=1}^{s-1}\left(k_{i} k_{i}^{\prime}\right)^{-1} .
$$

Observe that $r$ is fixed but that $s$ varies from term to term in the sum. The notation $i \in \Sigma$ is abusive. It is the inverval $I=\left[k_{i-1}, k_{i}\right]$ that belongs to $\Sigma$. Let $e_{I}=k_{i-1}, e_{I}^{\prime}=k_{i}^{\prime}$

The union of the intervals $I$ in $\Sigma$ may or may not cover $[0, k]$. There will be a certain number $g(\Sigma)$ of open intervals or gaps left uncovered. We divide the endpoints $\theta$ of the intervals in $\Sigma$ into four types, the extreme, the bound, the left free, the right free. The corresponding sets will be denoted $\Theta^{e}, \Theta^{b}, \Theta^{l f}, \Theta^{r f}$. The endpoint $\theta$ will be said to be extreme if it is 0 or $k$, to be left free if it separates a gap on its left from an interval of $\Sigma$ on its right, to be right free if it separates an interval on its left from a gap on its right, and to be bound if it is the common endpoint of two intervals in $I$.

Lemma 3.1. The coefficient $S_{k}^{r}(Y+1, \Delta)$ is the sum over all $\Sigma$ with $r$ elements of the product of

$$
(k+1)^{g(\Sigma)-2} \prod_{I \in \Sigma}\left(\Delta\left(Y+k+2-\left(e_{I}+1\right)\right)+(1-\Delta)\left(Y+e_{I}^{\prime}+1\right)\right)
$$

and

$$
\left\{\prod_{\boldsymbol{\Theta}^{e}} k \prod_{\boldsymbol{\Theta}^{b}} \theta(k-\theta) \prod_{\boldsymbol{\Theta}^{l f}}(k-\theta)(k+1-\theta) \prod_{\boldsymbol{\theta}^{r f}} \theta(\theta+1)\right\}^{-1} .
$$

The product in the first factor is obtained by writing

$$
k_{i}^{\prime}+Y+1+\Delta\left(k_{i-1}^{\prime}-k_{i}^{\prime}\right)=\Delta\left(Y+k+2-\left(e_{I}+1\right)\right)+(1-\Delta)\left(Y+e_{I}^{\prime}+1\right) .
$$

The factor $(k+1)^{-2}$ is extracted from the factor $(k(k+1))^{-2}$ in (2.a). Ignoring it, we have to evaluate the sum over all partitions containing a given $\Sigma$ of

$$
k^{-2}(k+1)^{s-r}\left(\prod_{i=1}^{s-1} k_{i} k_{i}^{\prime}\right)^{-1}
$$

The partitions containing $\Sigma$ are obtained by partitioning all the gaps. Thus the 
sum of the expressions (3.b) is the product of

$$
\left\{\prod_{\Theta^{e}} k \prod_{\Theta^{b}} \theta(k-\theta)\right\}^{-1}
$$

with the product over all gaps of

$$
\left(a(a+h) a^{\prime}\left(a^{\prime}+h\right)\right)^{-1} \sum_{A} \prod_{j \in A}(a+j)^{-1}\left(a^{\prime}+h-j\right)^{-1}\left(a+a^{\prime}+h+1\right)^{|A|+1} .
$$

Here $a$ is the left endpoint of the gap, $a^{\prime}$ is the distance of its right endpoint from $k$, and $h$ its length, so that $k=a+a^{\prime}+h$. If one of $a$ or $a^{\prime}$ is 0 , it is to be omitted from the factor preceding the sum, which runs over all subsets of $\{1, \ldots, h-1\}$.

The expansion (3.c) is the product of $k+1$ with

$$
\left(a(a+h) a^{\prime}\left(a^{\prime}+h\right)\right)^{-1} \prod_{j=1}^{h-1}\left(1+\frac{a+a^{\prime}+h+1}{(a+j)\left(a^{\prime}+h-j\right)}\right) .
$$

Upon simplification this becomes

$$
\left(a(a+h) a^{\prime}\left(a^{\prime}+h\right)\right)^{-1} \prod_{j=1}^{h-1} \frac{(a+j+1)\left(a^{\prime}+h-j+1\right)}{(a+j)\left(a^{\prime}+h-j\right)}=\frac{1}{a a^{\prime}} \cdot \frac{1}{(a+1)\left(a^{\prime}+1\right)} .
$$

The lemma follows. Notice that if, for example, $a=0$ then it is to be omitted, and $a+1=1$; so the right side of this equality is $1 / a^{\prime}\left(a^{\prime}+1\right)$.

The expression (2.b) is equal to

$$
\prod_{I}\left(\Delta\left(Y+k+2-e_{I}\right)+(1-\Delta)\left(Y+e_{I}^{\prime}\right)\right) \prod_{i=1}^{r+1}\left(k_{i} k_{i}^{\prime}\right)^{-1}
$$

The first product is over the intervals in the partitions. We expand this product by writing each of the linear factors in the first product as a sum of four terms: $\Delta(Y+k+2) ;-\Delta e_{I} ;(1-\Delta) Y ;(1-\Delta) e_{I}^{\prime}$. To these we attach respectively the labels: $(-1,0) ;(-1,1) ;(1,0) ;(1,1)$; or often, and preferably, the symbols: $\bullet ; \longleftarrow ;-$; $\longrightarrow$. The labels or the symbols are to be regarded as attached to the interval defining the linear factor, so that the label may be written $(\alpha(I), \mu(I))$. The first term defines the direction of the arrow. The whole collection of symbols defines a diagram

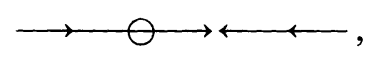

the lengths of the arrows being the lengths of the partitions. Let $a^{\prime}, a, b^{\prime}, b$ be the number of arrows of the four types, so that $a^{\prime}+a+b^{\prime}+b=r+2$. Then $P_{k+2}^{r+2}(Y, \Delta)$ may be written as

$$
\sum_{a^{\prime}, a, b^{\prime}, b}(-1)^{a} \Delta^{a^{\prime}+a}(1-\Delta)^{b^{\prime}+b}(Y+k+2)^{a^{\prime}} Y^{b^{\prime}} P_{k+2}\left(a^{\prime}, a, b^{\prime}, b\right) .
$$

The expression $P_{k+2}\left(a^{\prime}, a, b^{\prime}, b\right)$ is the sum over all diagrams (3.d) for which $a^{\prime}, a$, $b^{\prime}, b$ have the given values of

$$
\prod_{I} A(I)
$$

where

$$
A(I)=e_{I}^{\mu(I)-1} e_{I}^{\prime-1}
$$


if $I$ is negatively oriented, and

$$
A(I)=e_{I}^{\mu(I)-1} e_{I}^{-1}
$$

if it is positively oriented. It is again understood that $e_{I}$ or $e_{I}^{\prime}$ is suppressed if it is 0 . Moreover, those diagrams for which the initial interval is of type $\leftarrow$ or the final interval of type $\rightarrow$ are excluded from the sum.

It is clear from Lemma 3.1 that $S_{k}^{r}(Y+1, \Delta)$ has a similar expansion

$$
\sum_{a^{\prime}, a, b^{\prime}, b}(-1)^{a} \Delta^{a^{\prime}+a}(1-\Delta)^{b^{\prime}+b}(Y+k+2)^{a^{\prime}} Y^{b^{\prime}} S_{k}\left(a^{\prime}, a, b^{\prime}, b\right)
$$

where now the constraint on $a^{\prime}, a, b^{\prime}, b$ is that $a^{\prime}+a+b^{\prime}+b=r$. If any of $a^{\prime}, a, b^{\prime}, b$ is negative then $S_{k}\left(a^{\prime}, a, b^{\prime}, b\right)$ is understood to be 0 .

Observe that $(Y+\Delta)(Y+\Delta+k+1)$ is equal to the sum of

$$
\begin{gathered}
\Delta^{2}(Y+k+2)^{2}+2 \Delta(1-\Delta) Y(Y+k+2)+(1-\Delta)^{2} Y^{2}, \\
-\Delta(1-\Delta)(k+1)^{2}-\Delta(1-\Delta) Y(k+1)+\Delta(1-\Delta)(Y+k+2)(k+1),
\end{gathered}
$$

and

$$
-\Delta^{2}(Y+k+2)(k+1)+(1-\Delta)^{2} Y(k+1)
$$

while

$$
Y+(k+2) \Delta=\Delta(Y+k+2)+(1-\Delta) Y .
$$

Thus the identity is a result of the following lemma.

Lemma 3.2. $P_{k+2}\left(a^{\prime}, a, b^{\prime}, b\right)$ is equal to the sum of the four expressions:

and

$$
\begin{aligned}
& S_{k}\left(a^{\prime}-2, a, b^{\prime}, b\right)+2 S_{k}\left(a^{\prime}-1, a, b^{\prime}-1, b\right)+S_{k}\left(a^{\prime}, a, b^{\prime}-2, b\right) ; \\
& (k+1)^{2} S_{k}\left(a^{\prime}, a-1, b^{\prime}, b-1\right)+(k+1) S_{k}\left(a^{\prime}, a, b^{\prime}-1, b-1\right) \\
& \quad+(k+1) S_{k}\left(a^{\prime}-1, a-1, b^{\prime}, b\right) ; \\
& \quad(k+1) S_{k}\left(a^{\prime}-1, a, b^{\prime}, b-1\right)+(k+1) S_{k}\left(a^{\prime}, a-1, b^{\prime}-1, b\right) ;
\end{aligned}
$$

$$
(k+1) S_{k}\left(a^{\prime}, a, b^{\prime}-1, b\right)+(k+1) S_{k}\left(a^{\prime}-1, a, b^{\prime}, b\right) .
$$

If we ignore the length of the arrows in (3.d) we obtain diagrams in which only the direction and the type of arrow is significant. Given such a diagram, we let $P(*)$ be the sum over all partitions and labelings of type $*$ of $\prod_{I} A(I)$. To each diagram $*$ we can attach integers $a^{\prime}(*), a(*), b^{\prime}(*)$, and $b(*)$. Then

$$
P_{k+2}\left(a^{\prime}, a, b^{\prime}, b\right)=\sum P_{k+2}(*),
$$

the sum being over all diagrams with the given values of $a^{\prime}, a, b^{\prime}$, and $b$. For example, recalling the conditions on the extreme arrows, we see that

equals

$$
P_{k+2}(0,1,1,1)
$$

$$
P_{k+2}(\longrightarrow \mapsto \hookrightarrow \longleftarrow)+P_{k+2}(\longrightarrow \longleftarrow 1-\Theta)+P_{k+2}(\ominus \mapsto \longmapsto \longleftarrow) .
$$

In the same way,

$$
S_{k}\left(a^{\prime}, a, b^{\prime}, b\right)=\sum S_{k}(*)
$$




\section{A Special Case}

If $a^{\prime}=b^{\prime}=0$ then Lemma 3.2 reduces to

$$
P_{k+2}(0, a, 0, b)=(k+1)^{2} S_{k}(0, a-1,0, b-1) .
$$

This is implied immediately by the following proposition.

Proposition 4.1. If $a^{\prime}(*)=b^{\prime}(*)=0$ then

$$
P_{k+2}(\rightarrow * \leftarrow)=(k+1)^{2} S_{k}(*) .
$$

The abstract diagram $\rightarrow * \leftarrow$ is obtained by juxtaposing a sequence of diagrams of the form

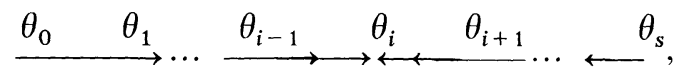

in which a sequence of positively directed arrows is followed by a sequence of negatively directed arrows. The contribution to $P(\rightarrow * \leftarrow)$ from a concrete diagram, one in which the endpoints of all arrows have been fixed, is the product over the diagrams (4.a) of

$$
\frac{1}{\theta_{0}} \frac{1}{\theta_{1}} \cdots \frac{1}{\theta_{i-1}}\left(\theta_{i+1}-\theta_{i-1}-1\right) \frac{1}{k+2-\theta_{i+1}} \cdots \frac{1}{k+2-\theta_{s-1}} \frac{1}{k+2-\theta_{s}} .
$$

Notice that $\theta_{i}$ does not occur here and the only condition on the sequence $\theta_{0}, \theta_{1}, \ldots, \theta_{i-1}, \theta_{i+1} \cdots \theta_{s-1}$ is that

$$
\theta_{0}<\theta_{1}<\cdots<\theta_{i-1}<\theta_{i+1}<\cdots<\theta_{s-1}<\theta_{s} .
$$

Notice that $1 / \theta_{0}$ is suppressed if $\theta_{0}=0$ and $1 / k+2-\theta_{s}$ is suppressed if $\theta_{s}=k+2$.

On the other hand, the abstract diagram $*$ begins with a sequence

$$
\rightarrow \cdots \rightarrow \text {, }
$$

that is then followed by a sequence of diagrams

and finally by a sequence

Any or all of these sequence may be empty.

The expression $(k+1)^{2} S_{k}(*)$ will be treated using Lemma 3.1. Since we have multiplied by $(k+1)^{2}$ the factor $(k+1)^{g(\Sigma)-2}$ may be replaced by $(k+1)^{g(\Sigma)}$.

There are two simple but important relations to note before beginning:

$$
\begin{aligned}
& \sum_{\theta<j<\eta} \frac{1}{(k-\theta) j} \cdot \frac{k+1}{(j+1)(k+1-\eta)}+\frac{1}{k-\theta} \cdot \frac{1}{\eta}=\frac{1}{(\theta+1)(k+1-\eta)} ; \\
& \sum_{\theta<j<\eta} \frac{1}{\eta(k-j)} \cdot \frac{k+1}{(k+1-j)(\theta+1)}+\frac{1}{(k-\theta) \eta}=\frac{1}{(\theta+1)(k+1-\eta)} .
\end{aligned}
$$


Since

$$
\frac{1}{j(j+1)}=\frac{1}{j}-\frac{1}{j+1}, \frac{1}{(k-j)(k-j+1)}=\frac{1}{k-j}-\frac{1}{k-j+1},
$$

the left-hand sides are easily calculated and found to be equal to the right-hand sides.

We next calculate a factor of that part of $S_{k}(*)$ obtained from diagrams whose first segment is

$$
\stackrel{\eta_{1} \quad \theta_{1}}{\longrightarrow} \stackrel{\eta_{s} \quad \theta_{s}}{\longrightarrow}
$$

with $\theta_{1}, \ldots, \theta_{s}$ given. Fixing $\theta_{1}$ and summing over the possible $\eta_{1}$, we obtain a contribution

$$
\left(k+1-\theta_{1}\right)\left\{\sum_{\eta_{1}=1}^{\theta_{1}-1} \frac{k+1}{\left(k+1-\eta_{1}\right)\left(k-\eta_{1}\right)} \cdot \frac{1}{\theta_{1}}+\frac{1}{k \cdot \theta_{1}}\right\}=1 .
$$

The factor $k+1-\theta_{1}$ comes from the product in the first term of Lemma 3.1. The factor $(k+1)$ in the numerator in the sum comes from the first gap, which is present if $\eta_{1}>0$ but not if $\eta_{1}=0$. The factor $1 / \theta_{1}$ is there whether $\theta_{1}$ is right free or bound. If $\theta_{1}$ is right free the additional factor $1 /\left(\theta_{1}+1\right)$ will be part of the contribution attached to the second arrow. The factor $\left(k+1-\eta_{1}\right)\left(k-\eta_{1}\right)$ is there because $\eta_{1}$ is left free if it is positive. If $\eta_{1}=0$ then it is extreme and contributes $1 / k$.

Passing on to the contribution from the second arrow, we fix $\theta_{1}$ and $\theta_{2}$, and form the sum,

$$
\left(k+1-\theta_{2}\right)\left\{\sum_{\eta_{2}=\theta_{1}+1}^{\theta_{2}-1} \frac{k+1}{\left(k+1+\eta_{2}\right)\left(k-\eta_{2}\right)} \cdot \frac{1}{\theta_{2}}+\frac{1}{\left(k-\theta_{1}\right) \theta_{2}}\right\}=\frac{1}{\theta_{1}+1} .
$$

Continuing, we obtain as the contribution from the initial segment, except for a supplementary factor arising from the last arrow that is to be treated later,

$$
\frac{1}{\theta_{1}+1} \cdots \frac{1}{\theta_{s-1}+1}
$$

where $0<\theta_{1}<\cdots<\theta_{s}$. In the same way the contribution of the final segment

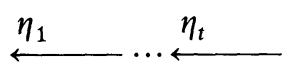

is

$$
\frac{1}{\left(k+1-\eta_{1}\right)} \cdots \frac{1}{\left(k+1-\eta_{t-1}\right)} .
$$

We now turn our attention to the contribution of one of the intermediate segments

First of all, fix $\theta, \eta$ and consider the total contribution of

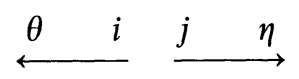


for all possible $i, j$. It is

$$
\frac{(\eta-\theta-1)}{(k-\theta) \eta}
$$

Once again, if $\theta$ is left free we do not include the factor $1 /(k+1-\theta)$, and if $\eta$ is right free we do not include the factor $1 /(\eta+1)$, nor do we include a factor $(k+1)$ from a gap at either end.

For a fixed $i$ and $j$ the contribution is

$$
\begin{array}{ll}
\frac{\theta+1}{k-\theta} \cdot \frac{1}{i(i+1)} \cdot \frac{1}{(k-j)(k+1-j)} \cdot \frac{(k+1-\eta)}{\eta} \cdot(k+1) & i<j, \\
\frac{\theta+1}{k-\theta} \cdot \frac{1}{i(k-j)} \cdot \frac{k+1-\eta}{\eta} & i=j .
\end{array}
$$

Fixing $j$ and summing over $i$ we obtain

$$
\sum_{\theta<j<\eta} \frac{1}{(k-j)} \cdot \frac{1}{(k+1-j)} \frac{k+1-\eta}{\eta}=\frac{(\eta-\theta-1)}{(k-\theta) \eta} .
$$

This is not a very useful form in which to have the contribution. To transform it, we prove some simple lemmas. To have some notation, set

$$
\begin{aligned}
f_{\theta}\left(\theta_{1}\right) & =\frac{k+1}{(\theta+1)\left(k+1-\theta_{1}\right)} & & \theta>\theta_{1}, \\
& =1 & & \theta=\theta_{1} .
\end{aligned}
$$

Set

$$
\begin{aligned}
g_{\eta}\left(\eta_{1}\right) & =\frac{k+1}{(k+1-\eta)\left(\eta_{1}+1\right)} & & \eta_{1}<\eta \\
& =1 & & \eta_{1}=\eta .
\end{aligned}
$$

Lemma 4.2. If $\theta$ and $\eta$ are given, $\theta<\eta$ then

is equal to

$$
\sum_{\theta \leq \theta_{1}<\eta_{1} \leq \eta} f_{\theta}\left(\theta_{1}\right) \frac{\left(\eta_{1}-\theta_{1}-1\right)}{\left(k-\theta_{1}\right) \eta_{1}} g_{\eta}\left(\eta_{1}\right)
$$

$$
\frac{1}{(\theta+1)(k+1-\eta)} \sum_{\theta<\theta_{2}<\eta} \frac{\theta_{2}\left(k-\theta_{2}\right)}{\left(\theta_{2}+1\right)\left(k+1-\theta_{2}\right)} .
$$

To calculate the sum over $\theta_{1}, \eta_{1}$ we write

$$
\eta_{1}-\theta_{1}-1=\left(k-\theta_{1}\right)+\eta_{1}-(k+1),
$$

obtaining three sums that we easily evaluate using (4.b) and (4.c).

$$
\begin{aligned}
& \sum_{\theta_{1}} \sum_{\eta_{1}} \frac{f_{\theta}\left(\theta_{1}\right) g_{\eta}\left(\eta_{1}\right)}{\eta_{1}}=\frac{1}{(\theta+1)(k+1-\eta)}\left\{\sum_{\theta<\theta_{1}<\eta} \frac{(k+1)\left(k-\theta_{1}\right)}{\left(k+1-\theta_{1}\right)\left(\theta_{1}+1\right)}+k-\theta\right\}, \\
& \sum_{\eta_{1}} \sum_{\theta_{1}} \frac{f_{\theta}\left(\theta_{1}\right) g_{\eta}\left(\eta_{1}\right)}{\left(k-\theta_{1}\right)}=\frac{1}{(\theta+1)(k+1-\eta)}\left\{\sum_{\theta<\eta_{1}<\eta} \frac{(k+1) \eta_{1}}{\left(k+1-\eta_{1}\right)\left(\eta_{1}+1\right)}+\eta\right\},
\end{aligned}
$$




$$
\begin{aligned}
&-\sum_{\theta_{1}} \sum_{\eta_{1}} \frac{f_{\theta}\left(\theta_{1}\right)(k+1) g_{\eta}\left(\eta_{1}\right)}{\left(k-\theta_{1}\right) \eta_{1}}=\frac{1}{(\theta+1)(k+1-\eta)} \\
& \cdot\left\{-\sum_{\theta<\theta_{1}<\eta} \frac{(k+1)^{2}}{\left(k+1-\theta_{1}\right)\left(\theta_{1}+1\right)}-(k+1)\right\} .
\end{aligned}
$$

Replace in all three sums on the right the variable of summation by $\theta_{2}$. Note also that

$$
\sum_{\theta<\theta_{2}<\eta} \frac{\left(k+1-\theta_{2}\right)\left(\theta_{2}+1\right)}{\left(k+1-\theta_{2}\right)\left(\theta_{2}+1\right)}=\eta-\theta-1=(k-\theta)+\eta-(k+1) .
$$

Then to complete the proof of the lemma, we have only to observe that

$$
(k+1)\left(k-\theta_{2}\right)+(k+1) \theta_{2}-(k+1)^{2}+\left(k+1-\theta_{2}\right)\left(\theta_{2}+1\right)=\theta_{2}\left(k-\theta_{2}\right) .
$$

Note that

$$
\sum_{\eta=\theta+1}^{\theta^{\prime}-1} \frac{(k-\theta)\left(k+1-\theta^{\prime}\right)}{(k+1-\eta)(k-\eta)}=\left(\theta^{\prime}-\theta-1\right)=\sum_{\eta=\theta+1}^{\theta^{\prime}-1} \frac{(\theta+1) \theta^{\prime}}{\eta(\eta+1)}
$$

Lemma 4.3. If $\theta$ and $\eta$ are given, $\eta>\theta$, and

$$
\begin{aligned}
& A=\sum_{\theta<\theta_{1}<\cdots<\theta_{s}<\eta} \frac{\eta \cdot\left(\theta_{1}-\theta-1\right)}{\theta_{1}} \cdot \frac{1}{\theta_{1}+1} \cdots \frac{1}{\theta_{s}+1}, \\
& B=\sum_{\theta<\theta_{1}<\cdots<\theta_{s}<\eta} \frac{1}{\theta_{1}+1} \cdots \frac{1}{\theta_{S}+1}\left(\eta-\theta_{s}-1\right),
\end{aligned}
$$

then $A=B$.

Set

$$
\begin{aligned}
& A^{\prime}=\sum_{\theta<\theta_{1}<\cdots<\theta_{s}<\eta} \frac{\eta(\theta+1)}{\theta_{1}} \cdot \frac{1}{\theta_{1}+1} \cdots \frac{1}{\theta_{s}+1}, \\
& B^{\prime}=\sum_{\theta<\theta_{1}<\cdots<\theta_{s}<\eta} \frac{1}{\theta_{1}+1} \cdots \frac{1}{\theta_{s-1}+1} .
\end{aligned}
$$

It suffices to show that $A^{\prime}=B$. Clearly

and

$$
B^{\prime}=\sum_{\theta<\theta_{1}<\cdots<\theta_{s-1}<\eta} \frac{1}{\theta_{1}+1} \cdots \frac{1}{\theta_{s-1}+1} \cdot\left(\eta-\theta_{s-1}-1\right),
$$

$$
A^{\prime}=\sum_{\theta<\theta_{2}<\cdots<\theta_{s}<\eta} \eta \cdot(\theta+1)\left(\frac{1}{\theta+1}-\frac{1}{\theta_{2}}\right) \frac{1}{\theta_{2}+1} \cdots \frac{1}{\theta_{s}+1} .
$$

Since

$$
\eta(\theta+1)\left(\frac{1}{\theta+1}-\frac{1}{\theta_{2}}\right)=\frac{\eta\left(\theta_{2}-\theta-1\right)}{\theta_{2}}
$$

the lemma follows by induction, for it is clearly true for $s=1$, when $B^{\prime}=$ $\eta-\theta-1=A^{\prime}$. 
Lemma 4.4. If $\theta$ and $\eta$ are given, $\eta>\theta$ and

$$
\begin{aligned}
& A=\sum_{\theta<\eta_{s}<\cdots<\eta_{1}<\eta} \frac{(k-\theta)\left(\eta-\eta_{1}-1\right)}{\left(k-\eta_{1}\right)} \frac{1}{k+1-\eta_{1}} \cdots \frac{1}{k+1-\eta_{s}}, \\
& B=\sum_{\theta<\eta_{s}<\cdots<\eta_{1}<\eta} \frac{1}{\left(k+1-\eta_{1}\right)} \cdots \frac{1}{\left(k+1-\eta_{s}\right)}\left(\eta_{s}-\theta-1\right),
\end{aligned}
$$

then $A=B$.

This is the previous lemma in another guise. Before we begin transforming the contributions of the intermediate segments, we treat the simplest case, that in which no intermediate sequence occurs in $*$. Then $\rightarrow * \leftarrow$ has concrete diagrams of the form

$$
\stackrel{\theta_{1}}{\longrightarrow} \ldots \stackrel{\theta_{s-1} \theta_{s}}{\longrightarrow} \stackrel{\theta_{s+1}}{\longleftrightarrow} \ldots \stackrel{\theta_{r+1}}{\longleftarrow}
$$

and $P(\rightarrow * \leftarrow)$ is the sum over $0<\theta_{1}<\cdots<\theta_{s-1}<\theta_{s+1} \cdots<\theta_{r+1}<k+2$ of

$$
\frac{1}{\theta_{1}} \cdots \frac{1}{\theta_{s-1}}\left(\theta_{s+1}-\theta_{s-1}-1\right) \frac{1}{k+2-\theta_{s+1}} \cdots \frac{1}{k+2-\theta_{r+1}} .
$$

The concrete diagrams of type $*$ with gaps are either of the form

$$
\stackrel{\theta_{1}}{\longrightarrow} \cdots \stackrel{\theta_{s-1}}{\longrightarrow} \stackrel{\theta_{s+1}}{\longleftarrow} \ldots \stackrel{\theta_{r+1}}{\longleftarrow},
$$

in which there is a gap between $\theta_{s-1}$ and $\theta_{s+1}$ or of the form

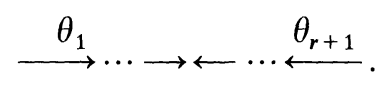

In the first case the supplementary factors arising from the two internal arrows are $1 /\left(\theta_{s-1}+1\right)$ and $1 /\left(k+1-\theta_{s+1}\right)$, together with a factor $k+1$ coming from the gap. This yields a contribution

$$
(k+1) \frac{1}{\theta_{1}+1} \cdots \frac{1}{\theta_{s-1}+1} \frac{1}{k+1-\theta_{s+1}} \cdots \frac{1}{k+1-\theta_{r+1}} \quad 0<\theta_{1}<\cdots<\theta_{r+1}<k .
$$

Substituting $\theta_{i}$ for $\theta_{i}+1$ in this product we see that we obtain all possible sequences contributing to (4.e) except those for which $\theta_{1}=1$ or $\theta_{r+1}=k+1$. Substracting a term (4.f) from the corresponding term of (4.e) we obtain

$$
\frac{1}{\theta_{1}} \cdots \frac{1}{\theta_{s-1}}\left(-\theta_{s-1}-\left(k+2-\theta_{s+1}\right)\right) \frac{1}{k+2-\theta_{s+1}} \cdots \frac{1}{k+2-\theta_{r+1}} .
$$

Breaking this into parts, cancelling either $\theta_{s-1}$ or $k+2-\theta_{s+1}$, and then taking account of the multiplicities with which the missing $\theta_{s-1}$ or $\theta_{s+1}$ could occur, we see that we have contributions

$$
\frac{-1}{1} \frac{1}{\theta_{1}} \cdots \frac{1}{\theta_{s-2}}\left(\theta_{s+1}-\theta_{s-2}-1\right) \frac{1}{k+2-\theta_{s+1}} \cdots \frac{1}{k+2-\theta_{r+1}}
$$


where

and

$$
0<1<\theta_{1}<\cdots<\theta_{s-2}<\theta_{s+1}<\cdots k+1
$$

$$
\frac{-1}{\theta_{1}} \cdots \frac{1}{\theta_{s-1}}\left(\theta_{s+2}-\theta_{s-1}-1\right) \frac{1}{\left(k+2-\theta_{s+2}\right)} \cdots \frac{1}{k+2-\theta_{r+1}} \frac{1}{(k+2)-(k+1)},
$$

where

$$
1<\theta_{1}<\cdots<\theta_{s-1}<\theta_{s+2}<\cdots<k+2 .
$$

This accounts for all contributions to $P(\rightarrow * \leftarrow)$ except those for which $\theta_{1}=1$, $\theta_{k+1}=k+1$.

However, concrete diagrams with no gap between $\theta_{s-1}$ and $\theta_{s+1}$ yield

$$
\frac{1}{\theta_{1}+1} \cdots \frac{1}{\theta_{s-2}+1} \frac{1}{k+1-\theta_{s+2}} \cdots \frac{1}{k+1-\theta_{r+1}} \text {. }
$$

Taking account of the multiplicity $\theta_{s+2}-\theta_{s-2}-1$ with which the missing $\theta_{s-1}=\theta_{s+1}$ can occur, we see that we obtain exactly those contributions to $P(\rightarrow * \leftarrow)$ associated to sequences $\theta_{1}=1, \theta_{r+1}=k+1$.

We now suppose that there is at least one intermediate segment, and treat the contribution of the first of these together with that of the initial segment. The junction of the two segments has the form

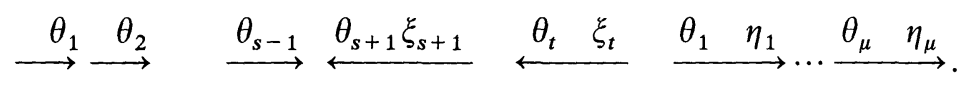

The arrow following upon $\eta_{\mu}$ is of the form $\leftarrow$. Suppose it begins at $\eta$.

If $u=1$ let $\eta^{\prime}=\eta$, but if $u>1$ we let $\eta^{\prime}=v_{2}$. If $t=s+1$, let $\theta^{\prime}=\theta_{s-1}$; otherwise let it be $\xi_{t-1}$. If the initial segment is empty, take $\theta^{\prime}=0$. In the same way let $\eta^{\prime}=k$ if it is otherwise undefined. Then Lemma 4.2 transforms the contribution of the interval $\left[\theta^{\prime}, \eta^{\prime}\right]$ to

$$
\sum_{\theta^{\prime}<\theta^{\prime \prime}<\eta^{\prime}} \frac{\theta^{\prime \prime}}{\theta^{\prime}+1} \cdot \frac{1}{\left(\theta^{\prime \prime}+1\right)\left(k+1-\theta^{\prime \prime}\right)} \cdot \frac{k-\theta^{\prime \prime}}{k+1-\eta^{\prime}} .
$$

The $\left(\theta^{\prime \prime}+1\right)\left(k+1-\theta^{\prime \prime}\right)$ in the denominator will not be touched. If $t>s+1$ we take $\theta^{\prime \prime} /\left(\theta^{\prime}+1\right)$ together with the rest of the contribution of the interval $\left[\theta_{t-1}, \xi_{t-1}\right]$ obtaining

$$
\frac{\theta^{\prime \prime}}{\xi_{t-1}+1} \cdot \frac{1}{\xi_{t-1}} \cdot \frac{\theta_{t-1}+1}{k-\theta_{t-1}}
$$

Summing over $\xi_{t-1}$ we obtain

$$
\frac{\theta^{\prime \prime}-\theta_{t-1}-1}{k-\theta_{t-1}}
$$

The contributions of the other intervals $\left[\theta_{i}, \xi_{i}\right], s+1 \leqq i<k-1$ then yield the sum of

$$
\sum_{\theta_{s 1}<\theta_{s+1}<\cdots<\theta_{t-1}<\theta^{\prime \prime}} \frac{\theta^{\prime \prime}-\theta_{t-1}-1}{k-\theta_{t-1}} \cdot \frac{1}{k-\theta_{t-1}+1} \cdots \frac{k+1}{k-\theta_{s+1}+1},
$$


and

$$
\sum_{\theta_{s-1}<\theta_{s+2}<\cdots<\theta_{t-1}<\theta^{\prime \prime}} \frac{\theta^{\prime \prime}-\theta_{t-1}-1}{k-\theta_{t-1}} \cdot \frac{1}{k-\theta_{t-1}+1} \cdots \frac{k+1}{k-\theta_{s+2}+1} \cdot\left(\theta_{s-1}+1\right) .
$$

The first factor is there so that in both cases we can use $1 /\left(\theta_{s-1}+1\right)$ as the contribution from the final arrow in the initial segment.

If $t-1$ is $s+1$, this manner of expressing the contribution is not obviously meaningful. It is better to take it as

$$
\sum_{\theta_{s-1}<\theta_{t-1}<\theta^{\prime \prime}}\left(\frac{\theta^{\prime \prime}-\theta_{t-1}-1}{k-\theta_{t-1}}\right)\left(\frac{k+1}{k+1-\theta_{t-1}}\right)+\left(\frac{\theta^{\prime \prime}-\theta_{s-1}-1}{k-\theta_{s-1}}\right) \cdot\left(\theta_{s-1}+1\right) .
$$

Lemma 4.5. The sum (4.j) is equal to

$$
\sum_{\theta_{s-1}<\theta_{t-1}<\theta^{\prime \prime}} \frac{\theta_{t-1}}{k+1-\theta_{t-1}} .
$$

Write the numerator in the first sum as

$$
(k+1)\left(k-\theta_{t-1}\right)-(k+1)\left(k+1-\theta^{\prime \prime}\right)
$$

The first term yields

$$
\sum_{\theta_{s-1}<\theta_{t-1}<\theta^{\prime \prime}} \frac{k+1}{k+1-\theta_{t-1}} .
$$

The second may easily be summed in closed form to yield

$$
-(k+1)\left(k+1-\theta^{\prime \prime}\right)\left(\frac{1}{k+1-\theta^{\prime \prime}}-\frac{1}{k-\theta_{s-1}}\right) .
$$

Adding to this the final term in (4.j) we obtain

$$
\left(-(k+1)\left(\theta^{\prime \prime}-\theta_{s-1}-1\right)+\left(\theta^{\prime \prime}-\theta_{s-1}-1\right)\left(\theta_{s-1}-1\right)\right) /\left(k-\theta_{s-1}\right)=-\left(\theta^{\prime \prime}-\theta_{s-1}-1\right)
$$

Since

$$
\theta^{\prime \prime}-\theta_{s-1}-1=\sum_{\theta_{s-1}^{\prime}<\theta_{t-1}<\theta^{\prime \prime}} \frac{k+1-\theta_{t-1}}{k+1-\theta_{t-1}}
$$

the lemma follows.

To deal with the case that $t-1>s+1$, we use Lemma 4.4 to write (4.h) as

$$
\sum_{\theta_{s-1}<\theta_{s+1}<\cdots<\theta_{t-1}<\theta^{\prime \prime}}(k+1)\left(\frac{\theta_{s+2}-\theta_{s+1}-1}{k-\theta_{s+1}}\right)\left(\frac{1}{k+1-\theta_{t-1}}\right) \cdots\left(\frac{1}{k+1-\theta_{s+1}}\right),
$$

and (4.i) as

$$
\sum_{\theta_{s-1}<\theta_{s+2}<\cdots<\theta_{t-1}<\theta^{\prime \prime}}\left(\frac{\theta_{s+2}-\theta_{s+1}-1}{k-\theta_{s+1}}\right)\left(\theta_{s-1}+1\right)\left(\frac{1}{k+1-\theta_{t-1}}\right) \cdots\left(\frac{1}{k+1-\theta_{s+2}}\right) .
$$

Applying Lemma 4.5 we obtain

$$
\sum_{\theta_{s-1}<\theta_{s+1}<\cdots<\theta_{t-1}<\theta^{\prime \prime}} \theta_{s+1}\left(\frac{1}{k+1-\theta_{t-1}}\right) \cdots\left(\frac{1}{k+1-\theta_{s+1}}\right) .
$$


Thus the conclusion is the same in all cases.

We put these contributions together with the contributions from the initial segment, writing $\theta_{s+1}$ as $\theta_{s+1}-\theta_{s-1}-1+\theta_{s-1}+1$. This yields a sum of two terms. The first is

$$
\frac{1}{\theta_{1}+1} \cdots \frac{1}{\theta_{s-1}+1}\left(\theta_{s+1}-\theta_{s-1}-1\right) \frac{1}{k+1-\theta_{s+1}} \cdots \frac{1}{k+1-\theta_{t-1}}
$$

the second, in which the multiplicity of the missing $\theta_{s-1}$ is taken into account, is

$$
\frac{1}{\theta_{1}+1} \cdots \frac{1}{\theta_{s-2}+1}\left(\theta_{s+1}-\theta_{s-2}-1\right) \frac{1}{k+1-\theta_{s+1}} \cdots \frac{1}{k+1-\theta_{t-1}} .
$$

Observe that upon replacing $\theta_{i}+1$ by $\theta_{i}$ these products correspond exactly to the initial contributions to $P(\rightarrow * \leftarrow)$, the second corresponding to $\theta_{1}=1$.

We now return to (4.g), and work towards the right; only the contribution $\left(k-\theta^{\prime \prime}\right) /\left(k+1-\eta^{\prime}\right)$ being pertinent. If there is only one intermediate segment, so that the complete diagram $*$ has the form

$$
\stackrel{\theta_{1}}{\longrightarrow} \cdots \stackrel{\theta_{s-1}}{\longleftrightarrow} \stackrel{\theta_{s+1}}{\longleftarrow} \stackrel{\theta_{t}}{\longleftarrow} \stackrel{\eta_{1}}{\longrightarrow} \stackrel{\eta_{u}}{\longrightarrow} \stackrel{\eta_{u+2}}{\longleftarrow} \cdots \stackrel{\eta_{r}}{\longleftrightarrow}
$$

then the contribution from the right is treated like that from the left and is the sum of

$$
\left(\frac{1}{\eta_{2}}\right) \cdots\left(\frac{1}{\eta_{u}+1}\right)\left(\eta_{u+2}-\eta_{u}-1\right)\left(\frac{1}{k+1-\eta_{u+2}}\right) \cdots\left(\frac{1}{k+1-\eta_{v}}\right)
$$

and

$$
\left(\frac{1}{\eta_{2}+1}\right) \cdots\left(\frac{1}{\eta_{u}+1}\right)\left(\eta_{u+3}-\eta_{u}-1\right)\left(\frac{1}{k+1-\eta_{u+3}}\right)\left(\frac{1}{k+1-\eta_{v}}\right) .
$$

The second corresponds to the contribution to $P(\rightarrow * \leftarrow)$ in which the final $\theta$ is $k+1$. Putting all contributions together, first multiplying the two parts and then adding over all possible sequences, and not forgetting $\left(\theta^{\prime \prime}+1\right)^{-1}\left(k+1-\theta^{\prime \prime}\right)^{-1}$ from (4.g) we see that we obtain $P(\rightarrow * \leftarrow)$.

Suppose, however, that there is a second intermediate segment, so that where they abut the diagram has the form

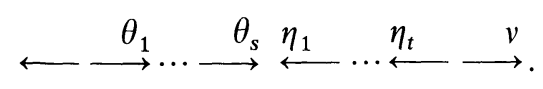

The contribution up to $\eta_{1}$ is calculated as before. For example, is $s=1 \mathrm{it}$, or rather the part coming from the right of $\theta^{\prime \prime}$, is

$$
\frac{k-\theta^{\prime \prime}}{k+1-\eta_{1}} \text {. }
$$

More generally, using Lemma 4.3 and the obvious modification of Lemma 4.5, 
we obtain

$$
\left(\frac{1}{\theta_{2}+1}\right) \cdots\left(\frac{1}{\theta_{s}+1}\right)\left(\frac{k-\theta_{s}}{k+1-\eta_{1}}\right)
$$

The product

$$
\left(\frac{1}{\theta_{2}+1}\right) \cdots\left(\frac{1}{\theta_{s}+1}\right)
$$

corresponds exactly to what we need for the comparison with the contributions to $P(\rightarrow * \leftarrow)$.

The final factor is to be put together with the contribution from $\eta_{1}$ on to yield

$$
\sum_{\theta_{s}<\eta_{1}<\cdots \eta_{t}<v}\left(\frac{k-\theta_{s}}{k+1-\eta_{1}}\right)\left(\frac{1}{k+1-\eta_{2}}\right) \cdots\left(\frac{1}{k+1-\eta_{t}}\right)\left(\frac{v-\eta_{t}-1}{\left(k-\eta_{t}\right) v}\right) .
$$

The final factor is the contribution previously calculated for a diagram $\leftarrow \rightarrow$. Lemma 4.4 replaces this with

$$
\left(\eta_{1}-\theta_{s}-1\right)\left(\frac{1}{k+1-\eta_{1}}\right) \cdots\left(\frac{1}{k+1-\eta_{t}}\right) \cdot \frac{1}{v} .
$$

The initial terms correspond exactly to what is needed for the comparison. Keep the final two for proceeding further to the right. Choose $\theta^{\prime}, \eta^{\prime}$ as for (4.g). Thus $\theta^{\prime}$ is either $\eta_{t-1}$ or $\theta_{s}$. Then we have a contribution

$$
\sum_{\theta^{\prime}<\eta_{t}<v \leqq \eta^{\prime}} \frac{1}{\left(k+1-\eta_{t}\right)} \frac{1}{v} f_{\eta^{\prime}}(v) .
$$

The sum is over $\eta_{t}$ and $v$.

Lemma 4.6. The sum $(4 . k)$ is equal to

$$
\sum_{\theta^{\prime}<\theta^{\prime \prime}<\eta^{\prime}} \frac{1}{\left(\theta^{\prime \prime}+1\right)\left(k+1-\theta^{\prime \prime}\right)} \cdot \frac{k-\theta^{\prime \prime}}{k+1-\eta^{\prime}} .
$$

This is an immediate consequence of (4.b). The factor $1 /\left(\theta^{\prime \prime}+1\right)\left(k+1-\theta^{\prime \prime}\right)$ is what we need for the comparison with the contribution to $P(\rightarrow * \leftarrow)$. The factor

$$
\frac{k-\theta^{\prime \prime}}{k+1-\eta^{\prime}}
$$

which we have seen before, is what we need to treat the second intermediate segment like the first, and then inductively to proceed all across the diagram, thereby proving the proposition.

\section{Completion of the Proof}

The proof of Lemma 3.2 will now be reduced to Proposition 4.1. Although the value of $P(*)$ or $S(*)$ depends on the directions of the uncircled areas in $*$ it does not depend on the directions of the circled arrows. They are important only when 
calculating $P_{k+2}\left(a^{\prime}, a, b^{\prime}, b\right)$ or $S_{k}\left(a^{\prime}, a, b^{\prime}, b\right)$. Thus we sometimes indicate a circled arrow simply by the symbol $\ominus$.

\section{Lemma 5.9.}

(a) $\quad P(-\bigcirc)=\frac{1}{k+1} P(\rightarrow \leftarrow)$,

(b) $\quad P(*-\bigcirc)=\frac{1}{k+1} P(* \rightarrow \leftarrow)+\frac{1}{k+1} P(* \leftarrow)$,

(c) $\quad P(-\bigcirc *)=\frac{1}{k+1} P(\rightarrow \leftarrow *)+\frac{1}{k+1} P(\rightarrow *)$,

(d) $P\left(*_{1}-*_{2}\right)=\frac{1}{k+1} P\left(*_{1} \rightarrow \leftarrow *_{2}\right)+\frac{1}{k+1} P\left(*_{1} \rightarrow *_{2}\right)+\frac{1}{k+1} P\left(*_{1} \leftarrow *_{2}\right)$,

(e) $S\left(*_{1}-\bigcirc *_{2}\right)=\frac{1}{k+1} S\left(*_{1} \leftarrow \rightarrow *_{2}\right)+\frac{1}{k+1} S\left(*_{1} \leftarrow *_{2}\right)+\frac{1}{k+1} S\left(*_{1} \rightarrow *_{2}\right)$.

In the formula (e) for $S$ both $*_{1}$ and $*_{2}$ may be empty, but in the formula (d) for $P$ they must not be, as must not be $*$ in formulas (b) and (c). Since $P(-\bigcirc-)=1$, $P(\rightarrow \leftarrow)=k+1$, the first formula is clear. Consider the second. The contribution of a concrete diagram

$\theta$

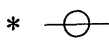

is of the form $A \cdot 1 / \theta$. The contribution of

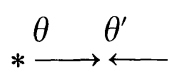

to $P(* \rightarrow \leftarrow)$ is $A \cdot(1 / \theta)(k+1-\theta)$, while that of

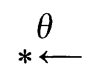

to $P(* \leftarrow)$ is $A$. The formula (c) is of course proved in exactly the same way. For (d) the verification is also similar. The diagram

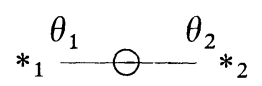

contributes a term $A \cdot\left(1 / \theta_{1}\right) \cdot\left(1 / k+2-\theta_{2}\right) \cdot B$. The contributions of the three diagrams appearing on the right are respectively:

$$
A \cdot \frac{1}{\theta_{1}} \cdot \frac{1}{k+2-\theta_{2}} \cdot\left(\theta_{2}-\theta_{1}-1\right) \cdot B ; \quad A \cdot \frac{1}{\theta_{1}} \cdot B ; \quad A \cdot \frac{1}{k+2-\theta_{2}} \cdot B .
$$

Proving the final formula is just a little less simple because we cannot operate entirely with concrete diagrams. The pertinent part of the concrete diagram for the left side of (e) is 


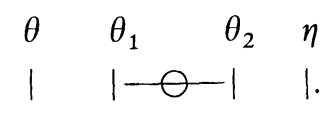

$\theta_{1}$ and $\theta_{2}^{*}$ are the endpoints of - and $\theta$ and $\eta$ are the closest endpoints of the arrows in $*_{1}$ or $*_{2}$. If $*_{1}$ is empty then $\theta=0$, and if $*_{2}$ is empty then $\eta=k$.

The contribution from the interior of the interval $[\theta, \eta]$ is

$$
\sum_{\theta \leqq \theta_{1}<\eta_{1} \leqq \eta} f_{\theta}\left(\theta_{1}\right) \cdot \frac{1}{\left(k-\theta_{1}\right) \eta_{1}} \cdot g_{\eta}\left(\eta_{1}\right) .
$$

By this we mean that the factors $1 / \theta$ and $1 /(k-\eta)$ are not taken into account.

On the other hand, replacing the undirected arrow - in the interval $\theta_{1}$, $\theta_{2}$ by the directed arrow $\rightarrow$, we obtain

$$
\sum_{\theta \leqq \theta_{1}<\eta_{1} \leqq \eta} f_{\theta}\left(\theta_{1}\right) \cdot \frac{k+1-\eta_{1}}{\left(k-\theta_{1}\right) \eta_{1}} g_{\eta}\left(\eta_{1}\right) .
$$

Taking instead $\leftarrow$, we obtain

$$
\sum_{\theta \leqq \theta_{1}<\eta_{1} \leqq \eta} f_{\theta}\left(\theta_{1}\right) \cdot \frac{\theta_{1}+1}{\left(k-\theta_{1}\right) \eta_{1}} g_{\eta}\left(\eta_{1}\right) .
$$

Next, inside the same interval $[\theta, \eta]$ we insert two opposing arrows

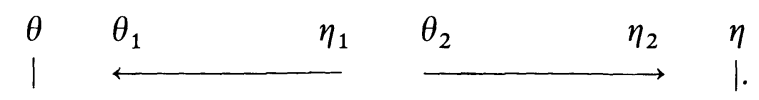

We have to take the sum

$$
\sum_{\theta \leqq \theta_{1}<\eta_{1} \leqq \theta_{2}<\eta_{2} \leqq \eta} f_{\theta}\left(\theta_{1}\right)\left(\frac{\theta_{1}+1}{\left(k-\theta_{1}\right) \eta_{1}}\right) g_{\theta_{2}}\left(\eta_{1}\right)\left(\frac{k+1-\eta_{2}}{\left(k-\theta_{2}\right) \eta_{2}}\right) g_{\eta}\left(\eta_{2}\right) .
$$

Use (4.b) to sum over $\eta_{1}$ to obtain

$$
\sum_{\theta \leqq \theta_{1}<\theta_{2}<\eta_{2} \leqq \eta} f_{\theta}\left(\theta_{1}\right)\left(\frac{1}{k+1-\theta_{2}}\right)\left(\frac{k+1-\eta_{2}}{\left(k-\theta_{2}\right) \eta_{2}}\right) g_{\eta}\left(\eta_{2}\right) .
$$

Then sum over $\theta_{2}$ to obtain

$$
\sum_{\theta \leqq \theta_{1}<\eta_{2} \leqq \eta} f_{\theta}\left(\theta_{1}\right) \frac{\eta_{2}-\theta_{1}-1}{\left(k-\theta_{1}\right) \eta_{2}} g_{\eta}\left(\eta_{2}\right) .
$$

The last formula of the lemma follows immediately on replacing $\eta_{2}$ in this sum by $\eta_{1}$, for

$$
\left(\theta_{1}+1\right)+\left(k+1-\eta_{1}\right)+\left(\eta_{1}-\theta_{1}-1\right)=k+1 .
$$

It is clear that the expansion permitted by this lemma can be iterated so that $P_{k+2}\left(a^{\prime}, a, b^{\prime}, b\right)$ becomes a weighted sum of $P(*)$, where $*$ is a diagram with no circled arrows, and with its extreme arrows pointing inwards. The diagrams that we obtain have distinguished subdiagrams of the type $\rightarrow \leftarrow, \rightarrow$, or $\leftarrow$, each provided with a supplementary orientation. Suppose there are $c, c_{+}, c_{-}$distinguished subdiagrams of the three types with negative orientation and $d, d_{+}, d_{-}$with positive 
orientation. Then $a^{\prime}=c+c_{+}+c_{-}, d^{\prime}=d+d_{+}+d_{-}$. In addition, $P(*)$ is to be provided with the weight $(k+1)^{-a^{\prime}-b^{\prime}}$. Since the weight is independent of $*$ it can often be ignored. Each $*$ that occurs is actually of the form $\rightarrow *_{1} \leftarrow$ with $a_{1}^{\prime}=a^{\prime}$, $b_{1}^{\prime}=b^{\prime}$. By Proposition 4.1 we may replace $(k+1)^{-a^{\prime}-b^{\prime}} P(*)$ by $(k+1)^{2-a^{\prime}-b^{\prime}} S\left(*_{1}\right)$.

There are many types of diagrams that contribute to the sum of Lemma 3.2, and it is best to introduce a supplementary lable, because the same diagram $*_{2}$ may contribute more than once. This supplementary label is one of the diagrams:

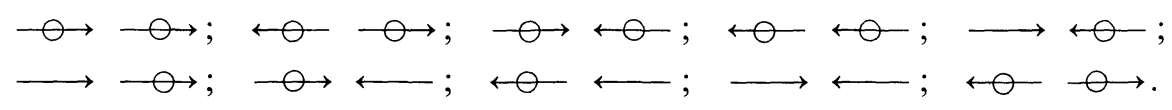

Each of the diagrams defines two numbers $e^{\prime}$, the number of negatively oriented circled arrows, and $f^{\prime}$, the number of positively oriented circled arrows. If $a_{2}^{\prime}=a^{\prime}\left(*_{2}\right), b_{2}^{\prime}=b^{\prime}\left(*_{2}\right)$ then $a_{2}^{\prime}+e^{\prime}=a^{\prime}, b_{2}^{\prime}+f^{\prime}=b^{\prime}$ and the weight attached to the contribution with the double label is $(k+1)^{2-e^{\prime}-f^{\prime}}$.

For example, the term $2 S_{k}\left(a^{\prime}-1, a, b^{\prime}-1, b\right)$ is the sum of contributions from pairs $\left(*_{2}, \bigcirc \leftrightarrow\right)$ and pairs $\left(*_{2}, \ominus \dashv\right)$, while $S_{k}\left(a^{\prime}, a, b^{\prime}-1, b\right)$ is the sum of contributions from pairs $\left(*_{2}, \bigcirc\right)$.

Using the lemma to expand, we obtain the common weight $(k+1)^{2-a^{\prime}-b^{\prime}}$. The terms of the expansion are labeled by the same supplementary diagram $*_{3}^{\prime}$, and by a diagram $*_{3}$ with no circled arrows but with a certain number of distinguished subdiagrams of the form $\leftarrow \rightarrow, \rightarrow$, or $\leftarrow$. Each of these subdiagrams is provided with an orientation, corresponding to the orientation of the circled arrow from which it arose. Let there be $i, i_{+}, i_{-}$negatively oriented arrows of the three types and $j, j_{+}, j_{-}$positively oriented. Then $a_{2}^{\prime}=i+i_{+}+i_{-}, b_{2}^{\prime}=j+j_{+}+j_{-}$.

We have to establish a bijection between the diagrams $*_{1}$, taken with multiplicity, and the diagrams $\left(*_{3}, *_{3}^{\prime}\right)$, also taken with multiplicity. Suppose $*_{1}$ is obtained from $*=\rightarrow *_{1} \leftarrow$. Thus $*$ is obtained by introducing one of the three diagrams $\rightarrow \leftarrow, \rightarrow, \leftarrow$, in place of each of the circled arrows. form

Let $D_{1}, \ldots, D_{s}$ be the introduced diagrams of the form $\rightarrow \leftarrow$, so that $*$ has the

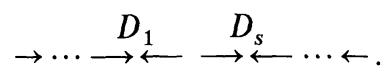

Thus there are $s+1$ sequences $S_{i}$ of arrows, from the beginning to the beginning of $D_{1}$, from the end of $D_{1}$ to the beginning of $D_{2}$, and so on. In $S_{i}, 1 \leqq i<s$, let there be $p_{i}$ positively directed arrows and $q_{i}$ negatively directed arrows. Let $p_{i}^{\prime}$ of the $p_{i}$ arrows arise from positively directed circled arrows by the substitution of $\rightarrow$ for $\circlearrowleft$, let $p_{i}^{\prime \prime}$ arise from negatively directed circled arrows, and let $p_{i}^{\prime \prime \prime}$ be original arrows. Define $q_{i}^{\prime}, q_{i}^{\prime \prime}, q_{i}^{\prime \prime \prime}$ similarly. Define $p_{s}, q_{s}, p_{s}^{\prime}, p_{s}^{\prime \prime}, p_{s}^{\prime \prime \prime}, q_{s}^{\prime}, q_{s}^{\prime \prime}, q_{s}^{\prime \prime \prime}$ in the same way, except that the two end sequences are put together for the counting. Then the multiplicity with which the diagram with given locations of the $D_{i}$ and given $S_{i}$, as well as given $p_{i}^{\prime}, p_{i}^{\prime \prime}, p_{i}^{\prime \prime \prime}, q_{i}^{\prime}, q_{i}^{\prime \prime}, q_{i}^{\prime \prime \prime}$ contributes is

$$
\prod_{i=1}^{s} \frac{p_{i} !}{p_{i}^{\prime} ! p_{i}^{\prime \prime} ! p_{i}^{\prime \prime \prime} !} \cdot \frac{q_{i} !}{q_{i}^{\prime} ! q_{i}^{\prime \prime} ! q_{i}^{\prime \prime \prime} !}
$$

Between the center of $D_{i}$ and that of $D_{i+1}, 1 \leqq i<s$ there is at least one diagram 
$\leftarrow \rightarrow$. Let the first be $E_{i}$. If there is such a diagram after $D_{s}$, let $E_{s}$ be the first. Consider the number of ways of assigning to the arrows between the centers of $D_{i}$ and $D_{i+1}$ that do not lie in $E_{i}$ both circles and circled directions, and in such a way that the total number of each type is as before. The number is

$$
\frac{p_{i} !}{p_{i}^{\prime} ! p_{i}^{\prime \prime} ! p_{i}^{\prime \prime \prime} !} \cdot \frac{q_{i} !}{q_{i}^{\prime} ! q_{i}^{\prime \prime} ! q_{i}^{\prime \prime \prime} !} \text {. }
$$

Notice that we have begun to describe the diagrams $*_{3}$ for which $E_{1}, \ldots, E_{s-1}$, and $E_{s}$ if it has been assigned, are the diagrams $\leftarrow \rightarrow$ introduced in the expansion.

If $E_{s}$ does not exist, then the multiplicity with which we can make the various assignments up to the center of $D_{1}$ and after the center of $D_{s}$ is

$$
\frac{p_{s} !}{p_{s}^{\prime} ! p_{s}^{\prime \prime} ! p_{s}^{\prime \prime \prime} !} \cdot \frac{q_{s} !}{q_{s}^{\prime} ! q_{s}^{\prime \prime} ! q_{s}^{\prime \prime \prime} !}
$$

Notice that the extreme arrows of $*$ no longer play a role; they are replaced by the first arrow of $D_{1}$ and the last of $D_{s}$.

To make the comparison, we note that if $E_{s}$ does not exist then $D_{s}$ is determined as the final subdiagram of the form $\rightarrow \leftarrow$. We also agree that $E_{i}$ and $D_{i}, 1 \leqq i<s$ are to be obtained from circled arrows with the same orientation. Moreover if $E_{s}$ does not exist, then we take $*_{3}^{\prime}$ to be $\Theta$ or $\Theta$, according to the circled orientation of the arrow yielding $D_{s}$. We finally note that $D_{i}$ is determined by $E_{i}$. We simply move to the left from $E_{i}$ to the first diagram $\rightarrow \leftarrow$.

Thus the bijection with multiplicity is established between diagrams $*_{1}$ in which $D_{s}$ is the final diagram $\rightarrow \leftarrow$ in $*$ and diagrams $\left(*_{3}, *_{3}^{\prime}\right)$ in which $*_{3}^{\prime}$ has only a single arrow.

If $D_{s}$ is not the final diagram $\rightarrow \leftarrow$ then $E_{s}$ exists, and we take it as coming from a circled arrow with the same orientation as $D_{s}$. The only difference in the counting of the multiplicities is that for the contribution to $S_{\mathrm{s}}$ we remove the arrows in $E_{s}$ as well as the extreme arrows and only add the first arrow of $D_{1}$ and the last of $D_{s}$. To make the count come out correctly, we adjoin the supplementary $*_{3}^{\prime}$ with two arrows, each of which has three states that can be interpreted as circled with positive orientation, circled with negative orientation, and uncircled. Observe that this argument is also valid for the case that $s=0$.

A pair of tables for the case $r=1, a^{\prime}=a=b=1, b^{\prime}=0$ may help. Diagrams with the same lable, which lies between (i) and (v), are matched in the bijection.

Initial

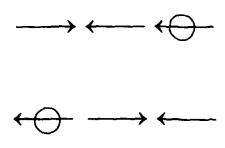

Expanded *

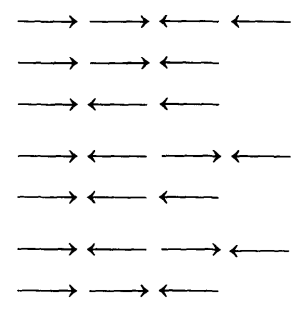

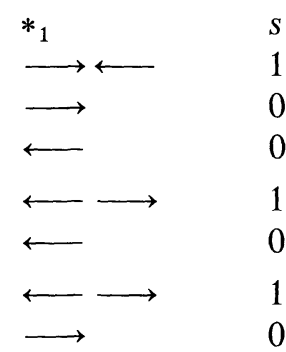



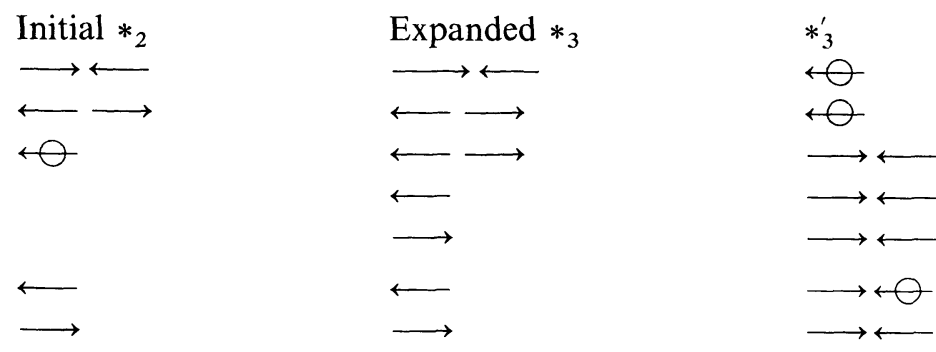

\section{Appendix}

The Virasoro algebra $\mathfrak{g}$ as an infinite-dimensional Lie algebra over $\mathbf{C}$ with basis $L_{k}, k \in \mathbb{Z}$, and $C$ and is defined by the conditions:

(i) $C$ is central;

(ii) $\left[L_{i}, L_{j}\right]=(i-j) L_{i+j}+\delta_{i,-j} \frac{i\left(i^{2}-1\right)}{12} C$.

The facts reviewed below can be found in [4].

The Verma modules are infinite-dimensional vector spaces $V_{h, c}$ on which $\mathfrak{g}$ acts and are attached to two real parameters $h$ and $c$. They are defined by the condition that they be universal with respect to the following property.

$V_{h, c}$ ix generated by a vector $v_{\phi}$ satisfying:

$$
C v_{\phi}=c v_{\phi}, \quad L_{o} v_{\phi}=h v_{\phi}, \quad L_{n} v_{\phi}=0, \quad n>0 .
$$

Thus $V_{h, c}$ has a basis

$$
v_{k_{1}, k_{2}, \ldots, k_{r}}=L_{-k_{1}} \cdots L_{-k_{r}} v_{\phi}, \quad k_{1} \geqq \cdots \geqq k_{r}>0 .
$$

However it may have other convenient bases as well. It carries a unique hermitian form $\langle u, v\rangle$ satisfying:

$$
\text { (i) }\left\langle v_{\phi}, v_{\phi}\right\rangle=1 \text {; } \text { (ii) }\left\langle L_{k} u, v\right\rangle=\left\langle u, L_{-k} v\right\rangle \text {. }
$$

Let $P(n)$ be the number of partitions of $n$. Then the space $V_{n}$ spanned by $\left\{v_{k_{1}, \ldots, k_{r}} \mid \sum k_{i}=n\right\}$ has dimension $P(n)$. If $\{$,$\} is the hermitian form defined by$ $\left\{v_{k_{1}, \ldots, k_{r}}, v_{l_{1}, \ldots, l_{s}}\right\}=0$ unless $r=s, k_{1}=l_{1}, \ldots, k_{r}=l_{r}$ then the matrix $H_{n}(h, c)$ of $\langle$,$\rangle on V_{n}$ with respect to $\{$,$\} is a polynomial in h$ and $c$ whose determinant is a constant times

$$
\prod_{p q \leqq n}\left(h-h_{p, q}\right)^{P(n-p q)} .
$$

If we introduce $m$ by the condition $c=1-6 / m(m+1)$, then

$$
h_{p, q}=h_{p, q}(m)=\frac{((m+1) p-m q)^{2}-1}{4 m(m+1)} \quad p, q \in \mathbb{N} .
$$

In particular if $n=p q$ then det $H_{n}$ vanishes on the curve $h=h_{p, q}(m)$. A null vector is obtained by taking some row $c_{k_{1}, \ldots, k_{r}}(m)$ of the matrix adjoint to $H_{n}$, 
and then setting

$$
w_{p, q}(m)=\sum_{k_{1}+\cdots+k_{r}=m} c_{k_{1}, \ldots, k_{r}}(m) v_{k_{1}, \ldots, k_{r}} .
$$

Choose the row so that the vector $\left(c_{k_{1}, \ldots, k_{r}}(m)\right)$ does not vanish identically. Since the coordinates of this vector are rational functions of $m$, we may write $\left(c_{k_{1}, \ldots, k_{r}}(m)\right)$ as the product of a rational function of $m$ and a vector $u_{p, q}(m)=\left(d_{k_{1}, \ldots, k_{r}}(m)\right)$ with coefficients that are polynomials in $m$ whose greatest common divisor is 1 . To stress the dependence on $p, q$, for it is important, I write

$$
d_{k_{1}, \ldots, k_{r}}(m)=d_{k_{1}, \ldots, k_{r}}^{p, q}(m) .
$$

To pass to a more convenient notation we take this $p, q$ to be $p^{\prime}, q^{\prime}$ and with another $p, q$ we set $\Delta=h_{p, q}(m)$. We form the rational functions of $m, Y, \Delta$ defined by

$$
a_{k_{1}, \ldots, k_{r}}(m, Y, \Delta)=\left(k_{r}+\cdots+k_{2}+y+\Delta k_{1}\right)\left(k_{r}+\cdots+k_{3}+Y+\Delta k_{2}\right) \cdots\left(Y+\Delta k_{r}\right)
$$

and by

$$
P_{p^{\prime}, q^{\prime}}(m, Y, \Delta)=\sum_{\substack{k_{1} \geqq \cdots \geq k_{r}>0 \\ k_{1}+\cdots+k_{r}=p^{\prime} q^{\prime}}} a_{k_{1}, \ldots, k_{r}}(m, Y, \Delta) d_{k_{1}, \ldots, k_{r}}^{p^{\prime}, q^{\prime}}(m) .
$$

The highest power of $Y$ that appears in $P_{p^{\prime}, q^{\prime}}(m, Y, \Delta)$ is $Y^{n}$, where $n=p^{\prime} q^{\prime}$, and it appears with coefficient $d_{1, \ldots, 1}^{p^{\prime}, q^{\prime}}(m)$.

On the other hand, set

$$
Y_{r, s}^{p^{\prime}, q^{\prime}}=h_{p^{\prime}, q^{\prime}}(m)-h_{p-r, q-s}(m) .
$$

Conjecture. If $\Delta=h_{p, q}$, then

$$
P_{p^{\prime}, q^{\prime}}(m, Y, \Delta)=d_{1, \ldots, 1}^{p^{\prime}, q^{\prime}}(m) \prod_{\substack{r+1 \equiv p^{\prime}(\bmod 2), r\left|<p^{\prime} \\ s+1 \equiv q^{\prime}(\bmod 2),\right| s \mid<q^{\prime}}}\left(Y-Y_{r, s}^{p^{\prime}, q^{\prime}}(m)\right) .
$$

It is important to observe that $p, q$ and $p^{\prime}, q^{\prime}$ play utterly different roles in this identity. Before explaining the significance of this conjecture, I observe that for generic $m$ the null space of $H_{n}(h, c), \quad h=h_{p^{\prime}, q^{\prime}}(m), c=c(m)$ is of dimension 1. Moreover, for all $k_{1}, \ldots, k_{r}$ such that $\sum k_{i}=n$ the vector

$$
v_{k_{1}, \ldots, k_{r}}=L_{-k_{1}} \cdots L_{-k_{r}} v_{\phi}
$$

lies in $V_{n}$, whether $k_{1}, \ldots, k_{r}$ are in decreasing order or not. In addition, it is not difficult to show that

$$
\sum_{\substack{k_{1}+\ldots+k_{r}=n \\ k_{1} \geq 0}} e_{k_{1}, \ldots, k_{r}} a_{k_{1}, \ldots, k_{r}}(m, Y, \Delta)
$$

depends only on the vector

Thus if

$$
\sum_{k_{1}+\cdots+k_{r}=n} e_{k_{1}, \ldots k_{r}} v_{k_{1}, \ldots, k_{r}} .
$$

$$
\sum e_{k_{1}, \ldots, k_{r}}(m) v_{k_{1}, \ldots, k_{r}}
$$


is any vector with values in the null space of $H_{n}(c, h)$ the function

$$
P(m, Y, \Delta)=\sum e_{k_{1}, \ldots, k_{r}}(m) a_{k_{1}, \ldots, k_{r}}(m, Y, \Delta)
$$

is determined by the coefficient $p(m, \Delta)$ of $Y^{n}$. Provided that this is not zero, the conjecture is tantamount to

$$
P(m, Y, \Delta)=p(m, \Delta) \prod_{r, s}\left(Y-Y_{r, s}^{p^{\prime}, q^{\prime}}(m)\right) .
$$

In the two extreme cases, $p^{\prime}=1$ or $q^{\prime}=1$, Benoit and Saint-Aubin [BSA] have found an explicit formula for $e_{k_{1}, \ldots, k_{r}}(m)$ :

a) $q^{\prime}=1$

$$
e_{k_{1}, \ldots, k_{r}}(m)\left(-\frac{(m+1)}{m}\right)^{n-r}\left\{\prod_{i=1}^{r-1}\left(k_{1}+\cdots+k_{i}\right)\left(k_{i+1}+\cdots+k_{r}\right)\right\}^{-1},
$$

b) $p^{\prime}=1$

$$
e_{k_{1}, \ldots, k_{r}}(m)\left(-\frac{m}{m+1}\right)^{n-r}\left\{\prod_{i=1}^{r-1}\left(k_{1}+\cdots+k_{i}\right)\left(k_{i+1}+\cdots+k_{r}\right)\right\}^{-1} .
$$

The conjecture in these extreme cases follows immediately from these formulas and Theorem 1.

The quotient of the Verma module $V_{h, c}$ by the space of null vectors is an irreducible module $M_{h, c}$. Select two parameters $\Delta^{\prime} \Delta^{\prime \prime}$, as well as a third parameter $\Delta$. A primary holomorphic field attached to these parameters is a formal series,

$$
\Phi(z)=z^{\Delta^{\prime \prime}-\Delta^{\prime}-\Delta} \sum_{k \in \mathbb{Z}} \varphi_{k} z^{k} .
$$

Here $\varphi_{k}$ is a matrix $\varphi_{k}=\varphi_{k, n^{\prime \prime}, n^{\prime}}, n^{\prime} \geqq 0, n^{\prime \prime} \geqq 0, n^{\prime}, n^{\prime \prime} \in \mathbb{Z}$ and

$$
\varphi_{k, n^{\prime \prime}, n^{\prime}}: M_{n^{\prime}}^{\prime} \rightarrow M_{n^{\prime \prime}}^{\prime \prime}
$$

We have set $M^{\prime}=M_{\Delta^{\prime}, c}, M^{\prime \prime}=M_{\Delta^{\prime \prime}, c} \cdot c=c(m)$, and

$$
M_{n^{\prime}}^{\prime}=\left\{v \in M^{\prime} \mid L_{o} v=\left(\Delta^{\prime}+n^{\prime}\right) v\right\}, \quad M_{n^{\prime \prime}}^{\prime \prime}=\left\{v \in M^{\prime \prime} \mid L_{o} v=\left(\Delta^{\prime \prime}+n^{\prime \prime}\right) v\right\} .
$$

Moreover $\varphi_{k, n^{\prime \prime}, n^{\prime}}=0$ unless $k=n^{\prime \prime}-n^{\prime}$; so we write $\varphi_{n^{\prime \prime}, n^{\prime}}$ for $\varphi_{n^{\prime \prime}-n^{\prime}, n^{\prime \prime}, n^{\prime}}$.

The formal series $\Phi(z)$ is said to define a primary holomorphic field of weight $\Delta$ if the identity

$$
L_{k} \Phi-\Phi L_{k}=z^{k+1} \frac{d}{d z} \Phi+\Delta(k+1) z^{k} \Phi
$$

is valid for all $k \in \mathbb{Z}$. This formal identity translates immediately into real identities for the operators $\varphi_{n^{\prime \prime}, n^{\prime}}$ between finite-dimensional vector spaces. Although it is usually ignored, a basic problem is to decide for what values of $\Delta^{\prime}, \Delta^{\prime \prime}$, and $\Delta$ a primary field exists. This is particularly important for the discrete series parameters $m \geqq 2, m \in \mathbb{Z}$,

$$
h=h_{a, b}(m) \quad 1 \leqq a<m, \quad 1 \leqq b<n+1 .
$$

Thus $\Delta^{\prime}, \Delta^{\prime \prime}, \Delta$ are all to belong to this set, $\Delta^{\prime}=h_{p^{\prime}, q^{\prime}}, \Delta^{\prime \prime}=h_{p^{\prime \prime}, q^{\prime \prime}}, \Delta=h_{p, q}$. 
It appears that it is not difficult to prove the existence of $\Phi(z)$ satisfying (A.a) provided one can construct $\varphi_{o, k}, \varphi_{k, o}$ for all $k \geqq 0$, satisfying the conditions implied by (A.a). It is moreover easy to see that $\varphi_{n^{\prime \prime}, n^{\prime}}$ is the adjoint of the operator $\varphi_{n^{\prime}, n^{\prime \prime}}$ attached not to $\Delta^{\prime}, \Delta^{\prime \prime}$ but to $\Delta^{\prime \prime}, \Delta^{\prime}$ and the same $\Delta$. Thus the necessary and sufficient condition for the existence of $\Phi(z)$ is that $\varphi_{o, k}$ exist for all $k$ for the parameters $\Delta^{\prime}, \Delta^{\prime \prime}$ and $\varphi_{o, k}$ for the parameters $\Delta^{\prime \prime}, \Delta^{\prime}$.

It is clearly enough to determine the conditions for the first problem. Normalize $\Phi$ so that $\varphi_{o, o}: v_{\phi}^{\prime} \rightarrow v_{\phi}^{\prime \prime}$. A simple calculation shows that

$$
\varphi_{o, k} v_{k_{1}, \ldots, k_{r}}^{\prime}=a_{k_{1}, \ldots, k_{r}}\left(m, \Delta^{\prime}-\Delta^{\prime \prime}, \Delta\right) v_{\phi}^{\prime \prime}, \quad k=\sum k_{i} .
$$

Thus a necessary and sufficient condition for the existence of $\varphi_{o, k}$ is that

imply that

$$
u=\sum c_{k_{1}, \ldots, k_{r}} v_{k_{1}, \ldots, k_{r}}^{\prime}=0
$$

$$
\sum c_{k_{1}, \ldots, k_{r}} a_{k_{1}, \ldots, k_{r}}\left(m, \Delta^{\prime}-\Delta^{\prime \prime}, \Delta\right)=0 .
$$

It will probably be easy to show that if the condition (A.b) is satisfied by one null vector, then it is satisfied for all null vectors obtained from it by repeatedly applying elements of the Virasoro algebra. After that, it should not be too difficult, using results of Feigin and Fuchs [FF], to show that for the discrete series it is enough to verify it for $u=u_{p^{\prime}, q^{\prime}}$ and $u=u_{m-p^{\prime}, m+1-q^{\prime}}$. Provided that $d_{1, \ldots, 1}^{p^{\prime}, q^{\prime}}(m)$ and $d_{1, \ldots, 1}^{m-p^{\prime}, m+1-q^{\prime}}(m)$ are not zero, and this seems likely, although I am not at the moment clear as to how it is to be proved, then the conjecture implies that the operators $\varphi_{o, k}$ exist for all $k$ if and only if

and

$$
\Delta^{\prime}-\Delta^{\prime \prime}=Y_{r_{1}, s_{1}}^{p^{\prime}, q^{\prime}}(m)
$$

$$
\Delta^{\prime}-\Delta^{\prime \prime}=Y_{r_{2}, s_{2}}^{m-p^{\prime}, m+1-q^{\prime}}(m),
$$

for some pairs $r_{1}, s_{1}, r_{2}, s_{2}$ satisfying $r_{1} \equiv p^{\prime}+1, \quad r_{2} \equiv m-p^{\prime}+1, \quad s_{1} \equiv q^{\prime}+1$, $s_{2} \equiv m-q^{\prime}$ modulo 2 , and $\left|r_{1}\right|<p^{\prime},\left|s_{1}\right|<q^{\prime},\left|r_{2}\right|<m-p^{\prime},\left|s_{2}\right|<m+1-q^{\prime}$.

For the present it is sufficient to examine the first equation, our only purpose being to verify that it leads to explicit conditions on $p, q, p^{\prime}, q^{\prime}$, and $p^{\prime \prime}, q^{\prime \prime}$. Set $p_{o}=p-r_{1}, q_{o}=q-s_{1}$. Since

$$
\Delta^{\prime}-\Delta^{\prime \prime}=h_{p^{\prime}, q^{\prime}}-h_{p^{\prime \prime}, q^{\prime \prime}}
$$

we infer from (A.c) that

$$
\left(p^{\prime \prime}-q^{\prime \prime}\right)^{2} m^{2}+2\left(p^{\prime \prime}+q^{\prime \prime}\right) p^{\prime \prime} m+p^{\prime \prime 2}=\left(p_{o}-q_{o}\right)^{2} m^{2}+2\left(p_{o}-q_{o}\right) p_{o} m+p_{o}^{2} .
$$

This equation is readily seen to be equivalent to the condition that either

or

$$
\left(p^{\prime \prime}-q^{\prime \prime}\right)-\left(p_{o}-q_{o}\right) m+\left(p^{\prime \prime}-p_{o}\right)=\left(p^{\prime \prime}-p_{o}\right)(m+1)-\left(q^{\prime \prime}-q_{o}\right) m=0
$$

$$
\left(p^{\prime \prime}-q^{\prime \prime}\right)+\left(p_{o}-q_{o}\right) m+\left(p^{\prime \prime}+p_{o}\right)=\left(p^{\prime \prime}+p_{o}\right)(m+1)-\left(q^{\prime \prime}+q_{o}\right) m=0 .
$$

The second equation is obtained from the first on substituting $p^{\prime \prime} \rightarrow m-p^{\prime \prime}, q^{\prime \prime} \rightarrow$ $m+1-q^{\prime \prime}$.

Since all quantities involved are integers, all solutions of the first equation are of the form $p^{\prime \prime}=p_{o}+a m, q^{\prime \prime}=q_{o}+a(m+1), a \in \mathbb{Z}$. Since $0<p, p^{\prime}, p^{\prime \prime}<m, 0<q$, 
$q^{\prime}, q^{\prime \prime}<(m+1)$, the integer a must be $0, \pm 1$ although not all these values are necessarily allowed.

The conclusion is that the conjecture leads to explicit, albeit somewhat elaborate, necessary and sufficient conditions on $p, q, p^{\prime}, q^{\prime}, p^{\prime \prime}, q^{\prime \prime}$ for the existence of a primary conformally invariant holomorphic field. There is a close connection between the problems of existence and the problem of establishing fusion rules. The conditions derived from the conjecture are close to those the attentive reader of the discussion of fusion rules in [BPZ] would expect, although perhaps not exactly the same.

\section{References}

1. Belavin, A. A., Polyakov, A. M., Zamolodchikov, A. B.: Infinite conformal symmetry in twodimensional quantum field theory. Nucl. Phys. B241, 333-380 (1984)

2. Benoit, L., Saint-Aubin, Y.: Explicit expressions for the null vectors $\varphi_{p, 1}$ and $\varphi_{1, q^{\prime}}$ of the discrete series representations of the Virasoro algebra. Phys. Lett. B215, 517-522 (1978)

3. Feigin, B. L., Fuchs, D. B.: Verma modules over the Virasoro algebra. In: Topology, SLN 1060 (1982)

4. Kac, V., Raina, A.: Lectures on highest-weight representations of infinite-dimensional Lie algebras. Singapore: World Scientific 1987

Communicated by A. Jaffe

Received October 6, 1988; in revised form November 28, 1988

Note added in proof. After this paper was submitted two preprints came to my attention that render the combinatorial arguments in it superfluous from some points of view, although not necessarily redundant. First of all, G. Felder in BRST Approach to Minimal Models has used the BRST method to construct primary fields and the conjecture of the appendix follows readily from his conclusions. Moreover, B. L. Feigin and D. B. Fuchs in Cohomology of some nilpotent subalgebras of the Virasoro and Kac-Moody Lie algebras take the direct algebraic approach of the appendix to the existence of primary fields, but deal with the whole matter much more efficiently, more generally, and more elegantly. It is in particular clear from their discussion that the conjecture is an easy consequence of results already in [3]. 